\title{
SCRITTI GIOVANILI DI ROBERTO DE VISIANI DALMATA SIBENICENSIS
}

\author{
Boško Knežić, Università di Zara, boknezic@unizd.hr
}

Original scientific paper

DOI: 10.31902/fll.34.2021.10

UDC: 78:929 DE VIZIANI R.

Con il presente articolo ci si prefigge di dare una visione dei primi componimenti giovanili inediti dell'illustre botanico Roberto de Visiani, conservati nell'Archivio storico dell'Orto botanico di Padova, nel faldone n. 36, fascicolo n. 5. Si tratta di manoscritti rilegati assieme in cartoncino, per la maggior parte scritti tra il 1817 e il 1818, dal titolo De Visiani. Scritti giovanili. Il contenuto del faldone si può dividere, grosso modo, in scritti compiuti (alcuni riportanti i titoli e altri senza titoli), e in scritti incompiuti, presentati in forma di carte sciolte destinate a ritocchi. L'attenzione sarà incentrata principalmente sui componimenti poetici compiuti e privi di autocorrezioni e annotazioni varie. Si tratta di testi d'occasione scritti in conformità alla poetica dei tempi in cui sono nati.

Parole chiave: Roberto de Visiani, Scritti giovanili, Archivio storico dell'Orto botanico di Padova

\section{Introduzione}

Il futuro professore di botanica dell'Università di Padova nacque a Sebenico, il 9 aprile $1800^{1}$ nella famiglia del medico Giovanni Battista ${ }^{2}$ e

${ }^{1}$ Si veda Libro degli atti di nascita Sebenico - Città (1737-1812), p. 102.

${ }^{2}$ La famiglia de Visiani si trasferì dall'Italia in Dalmazia verso la prima metà del Settecento, stabilendosi prima a Spalato da cui nel 1765 uno dei discendenti, Antonio Roberto, si trasferì a Sebenico dove nel 1768 aprì una farmacia. Il figlio di Antonio Roberto, Giovanni Battista, laureatosi in medicina all'Università di Padova nel 1794, tornò a Sebenico, dove nel 1807 assunse l'incarico di direttore del nuovo ospedale civico. Durante il suo soggiorno a Sebenico nel 1863, suo figlio Roberto de Visiani fece innalzare l'ospedale di un altro piano, mettendo a disposizione una somma di 2.800 fiorini, come testimonia la lapide affissa al muro dell'ospedale, ancora oggi visibile: A - Roberto de Visiani - Botanico Illustre Celeberrimo - Che - Questo Civico Spedale - Nel MDCCCVII - Per Solerzia di Suo Padre Inaugurato - Consorse Ad Aggradire E Rinnovare - Nel MDCCCLXIII - La Patria. Cfr. Grubišić 1983, 30-34 e Stošić 2006, 20. Dopo la costruzione del nuovo ospedale provinciale, avvenuta nel 1883 per volontà della Dieta dalmata, l'edificio fu modificato in ospizio dei poveri. A de Visiani non furono estranei gli atti di beneficenza che destinava alla città natale ed ai 
di Maddalena Drassich. La data di nascita di de Visiani fu, però, oggetto di leggeri disaccordi, così in alcuni scritti e articoli in memoriam apparsi dopo la scomparsa dell'illustre botanico si riporta il 3 marzo 1800 come data di nascita. A presentare dati che si rivelano in chiaro contrasto con quanto annotato nel Libro degli atti di nascita è stato Paolo Mazzoleni ${ }^{3}$, ospite di de Visiani a Padova nei primi giorni del 1878. In seguito alla richiesta di Mazzoleni di raccontare alcuni dati della sua vita, de Visiani gli consegnò un autografo nel quale, tra l'altro, si legge: "Roberto de Visiani nacque in Sebenico nella Parrocchia di S. Trinità il 3 marzo $1800^{4}$ di Giovanni Battista, medico in quella città, e di Maddalena Drassich» (cfr. Mazzoleni 1900, 17). La questione è stata risolta grazie ai due documenti autografi conservati presso la Biblioteca dell'Orto botanico di Padova, risalenti all'anno 1835 ed all'anno 1877, che confermano l'esattezza delle informazioni riportate nel Libro degli atti di nascita, togliendo ogni dubbio sulla data di nascita di de Visiani (cfr. Ibidem) Terminati gli studi elementari nella città natale dove ebbe come maestro fra' Antonio Tommaseo, de Visiani si recò a Spalato dove nel famoso Seminario strinse amicizia con Niccolò Tommaseo che sarebbe diventato uno dei suoi migliori amici e corrispondenti. Nel 1817 si trasferì a Padova dedicandosi allo studio di medicina, che portò a termine con successo nel 1822. Lo stesso anno il prof. Giuseppe Bonato lo propose come suo assistente, la cui nomina avvenne a seguito di un concorso con Decreto del governo di Venezia. Dal 1827 al 1835 alla fine del suo assistentato, esercitò la professione di medico a Cattaro (1829), Dernis (1830-1835) ed infine a Budua (1835). Nel 1836 de Visiani tornò a Padova, dove fu chiamato a sostituire il prof. Bonato in qualità di supplente, professore e prefetto dell'Orto botanico. Dopo gli esami di concorso alla cattedra di Botanica sostenuti a Vienna, nel $1837 \mathrm{fu}$ confermato stabilmente nel doppio incarico di professore ordinario e prefetto dell'Orto botanico, posizione che mantenne fino al 1877

suoi abitanti. Tra le donazioni disposte a favore della città di Sebenico, Paolo Mazzoleni registra un dono di libri per la biblioteca delle scuole, il ritratto di Niccolò Tommaseo con l'iscrizione "Questa effigie / Del maggiore de' suoi figli / Donava alla patria / Roberto de Visiani», seicento fiorini per l'ospedale e quattrocento fiorini destinati ai poveri della parrocchia in cui nacque. Cfr. Mazzoleni 1880, 6-7.

${ }^{3}$ Per ulteriori informazioni su Paolo Mazzoleni si rinvia a Knežić 2016, 197-209.

${ }^{4}$ Anche Tommaseo nutriva alcuni dubbi intorno alla sua data di nascita, così il 4 ottobre 1833 tracciò nel suo diario: «Mio giorno natalizio». Più tardi aggiunse in margine: "Sbaglio: il dì 8». Cfr. Tommaseo 1938, 135. La data annotata nel Libro degli atti di nascita (1788-1812) della Parrocchia di San Giacomo di Sebenico a pagina 127 è il 9 ottobre. 
quando il suo assistente, il prof. Pier Antonio Saccardo, lo sostituì alla cattedra (cfr. Notolini 2017-2018, 41-44). Tutta la sua vita, de Visiani la trascorse a Padova dove, il 4 maggio 1878 moriva senza moglie né prole. A Paolo Mazzoleni, al dott. Luigi Zuliani e al vescovo di Sebenico Antonio Fosco fu affidato l'adempimento delle sue ultime volontà, nelle quali disponeva «che la sua salma si trasportasse in patria, amando riposare tra i suoi congiunti amici e concittadini» (Mazzoleni 1900, 11). Due anni dopo la morte, le sue spoglie furono trasportate a Sebenico dove, grazie ad un supporto finanziario offerto dall'Università di Padova, nel cimitero di Sant'Anna gli fu innalzato un monumento sepolcrale ${ }^{5}$ inaugurato il 10 luglio 1880 (cfr. Mazzoleni 1900, 11).

\section{De Visiani poeta}

Nonostante avesse dedicato l'intera sua vita agli studi di botanica, de Visiani, discepolo del Seminario di Spalato noto per la coltivazione di lettere classiche, non si limitò a studiare soltanto il mondo della natura, ma si immerse con la stessa passione negli studi letterari e linguistici e in ciò l'amico Tommaseo gli fu principale critico e revisore. Mi sia permesso non dedicare in questa sede la dovuta attenzione a tutte le pubblicazioni con le quali l'illustre botanico contribuì all'arricchimento delle conoscenze scientifiche nel campo delle scienze naturali, lasciando che chi se ne intende esprima un giudizio ${ }^{6}$.

5 Il monumento visibile ancora oggi, opera di Domenico Pasini, è arricchito dall'epigrafe commemorativa stilata da Mauro Ricci: Heic ubi praeoptaverat Inter gentiles - Et municipes suos - Compositus est - Robertus de Visiani - An. natos LXXVIII - Qui medicinam professus - Patavii ubi in lyceo magno - Per an. XLIV - Rem herbariam tradidit - IV. non. maias an. MDCCCLXXVIII. - Decessit Doctrina scriptisque clariuimus - Flora Dalmatica - Insignem elenchum Aliaque egregia volumina - Edidit - Christiane sapientiae - Sectator - In publicum valetudinarium - In egenorum solatium - Benevolentia eluxit. Cfr. Stošić 2006, 20.

${ }^{6}$ Oltre a dedicarsi allo studio della flora della Dalmazia, per lui fonte ispiratrice sin dalla più tenera età, de Visiani fu affascinato dalla flora dell'Egitto, della Nubia, della Grecia e dell'Asia minore, nonché della flora fossile. Studiando la flora dei paesi vicini, scoprì e descrisse alcune piante rare della Serbia, della Bosnia e del Montenegro. Nel 1978 correva il centesimo anniversario della morte dell'illustre botanico e in quell'occasione non mancarono l'opera e l'autorità del Comune di Sebenico ad onorarlo. Sotto gli auspici dell'Accademia jugoslava di scienze, lettere ed arti, dal 5 all'8 ottobre fu organizzato in quella città un convegno internazionale dedicato alla vita e all'opera di Roberto de Visiani. II convegno, che vide più di 50 partecipanti dall'Italia e dall'allora Jugoslavia, si chiuse in maniera solenne con l'inaugurazione della lapide 
Sebbene gli ultimi decenni della sua vita venissero considerati il periodo in cui intensificò la sua attività di scrittore ${ }^{7}$, de Visiani già nel 1847, incoraggiato dal Tommaseo ${ }^{8}$, pubblicava sulla "Strenna dalmata ${ }^{9}$ un breve resoconto del suo viaggio lungo la costa dalmata, intitolato Una corsa botanica sul battello a vapore lungo la costa della Dalmazia. Scritta in maniera raffinata, con una cura particolare all'espressione ed al linguaggio, la breve descrizione della «tiepida e variata spiaggia della Dalmazia» da Zara a Cattaro ${ }^{10}$ con brevi soste a Sebenico, Scardona,

commemorativa (o ripristinamento, visto che la lapide inizialmente inaugurata nel 1890 fu scalpellata dopo la Seconda guerra mondiale) sulla casa natale di de Visiani. Quello stesso anno, nella traduzione croata di Jure Radić e con prefazione di Petar Matković, vide la luce la sua prima opera Stirpium Dalmaticarum Specimen contenente l'intera sua bibliografia.

7 Nel 1864 de Visiani pubblica in "La Gioventù» Accenni alle cognizioni botaniche di Dante nella Divina Commedia, scritto ripubblicato l'anno seguente in Dante e il suo secolo, Firenze, Coi tipi di M. Cellini sotto il titolo Accenni alle scienze botaniche nella Divina Commedia. Seguono poi i due volumi dell'opera di Valerio Massimo De' fatti e detti degni di memoria della Città di Roma e delle stranie genti: testo di lingua del secolo XIV riscontrato su molti codici e pubblicato da Roberto de Visiani (1867-1868), Del Tesoro volgarizzato di Brunetto Latini, edito sul più antico dei codici, raffrontato con più altri e col testo originale francese (1869) e altri.

${ }^{8}$ Nella lettera del 10 dicembre 1846 Tommaseo, tra l'altro, scrive a de Visiani: "Grazie di quel che mandasti alla Strenna, e le sarà, credo, il miglior ornamento". Cfr. Lettera inedita da Tommaseo a de Visiani, Biblioteca dell'Orto botanico dell'Università di Padova, Ar.B.13.

9 "Strenna Dalmata» fu il supplemento al settimanale «La Dalmazia» che fu pubblicato a Zara dal 1845 al 1847. L'annuario uscì a gennaio 1847, e fu annunciato all'ultima pagina del numero 48, del 26 novembre 1846, de «La Dalmazia»: «Ogni provincia, anzi ogni colta città quasi, suole offrire a' suoi cari nel rinnovarsi dell'anno od in altra principale festività una Strenna, in cui raccogliendo le più gradevoli produzioni intellettuali della patria, procura loro utile trattenimento. Finora simile lodevole costume non si tenne in Dalmazia, ed a noi venne pensiero di tentarlo, stampando una Strenna Dalmata, e la generosa condiscendenza di parecchi pregevolissimi scrittori nazionali, alcuni de'quali sono: Fenzi Antonio, Foscolo de Giorgio, Nisiteo Pietro, Paravia cav. P. Aless., Solito, Tommaseo D.r Niccolò ecc. che ci onorano di più loro inediti scritti, ne pongono al caso di farlo con felice riuscita». Per maggiori informazioni si veda Tomas 2018 .

10 «Dopo una breve corsa da Ragusa il piroscafo entra nell'ameno e tortuoso canale di Cattaro, fiancheggiato d'ambe le sponde da miti colli rallegrati da perpetua verzura. In questi e sul monte Sella, che domina la città, crescono di belle piante fra le quali si vantaggiano per rarità la Stachys menthaefolia, Picris laciniata, Delphinium divaricatum, Ophrys bonbylifora, Echium petraeum, 
Spalato, Salona, Traù, Lesina, Ragusa e Curzola, oltre ad essere un bell'esempio di prosa scientifica ${ }^{11}$ che presta pari attenzione sia alla dignità stilistica del testo che al contenuto scientifico, rappresenta anche un tentantivo abbastanza riuscito di un testo di viaggio indirizzato ad un pubblico selezionato:

Di quest'ultima fatta di viaggiatori i botanici sono forse i più numerosi, e siccome anche fra questi ve n'hanno o ne possono essere di sì frettolosi da contentarsi di visitare a sola costa, passandovi e ripassandovi col piroscafo così m'è paruto non disutile il farmi a questi, se non guida, compagno, additando loro quel meglio, che in fatto di piante posseggono i varii siti in cui fa sosta il battello che li conduce (De Visiani 1847, 82).

Il particolare stile scientifico di de Visiani, stilisticamente equilibrato e permeato di locuzioni poetiche, deve molto ai suoi primi componimenti giovanili rimasti inediti, conservati nell'Archivio storico dell'Orto botanico di Padova nel faldone n. 36, fascicolo n. $5^{12}$. Si tratta

Farsetia mutabilis, e fin sui tetti s'abbarbica agli embrici e cresce gigante il Saseli globiferum agitando all'aria i penduli globi, fitti e gremiti di fiorellini e di semi» (De Visiani 1847, 86).

${ }^{11}$ Molti sono i testi usciti dalla penna di de Visiani dedicati ai temi botanici, ai quali egli approda servendosi di uno stile elevato, prestando particolare attenzione alla scelta dei vocaboli, nonché alla composizione ritmica e stilistica della frase. Ai titoli già menzionati aggiungo Della utilità ed amenità delle piante, Discorso inaugurale detto il dì XX aprile dell'anno MDCCCXXXVII nella grande aula della I. R. Università di Padova da Roberto de Visiani, professore di botanica, Coi tipi della Minerva, Padova, 1837 e Delle benemeranze dei Veneti: discorso letto nella sala de' pregadi nel Palazzo Ducale in Venezia nel dì 30 maggio 1854.

12 L'autore ringrazia vivamente la disponibilità della Dott.ssa Paola Mario e del Dott. Moreno Clementi della Biblioteca dell'Orto botanico dell'Università di Padova. Il contenuto del faldone è stato brevemente descritto da Cvjetko Mika nell'articolo pubblicato negli Atti del convegno dedicato al centenario della morte di de Visiani, tenutosi nel 1978 a Sebenico. Mika tralascia di menzionare tutti gli scritti raccolti nel faldone, il che non stupisce visto che si tratta di carte maggiormente sciolte e numerate in una maniera che rende difficile seguire un ordine preciso. Mika dedica due pagine all'analisi della poesia Congedo dalla Dalmazia, mentre gli altri scritti del faldone vengono semplicemente elencati e sinteticamente descritti: il primo componimento senza titolo verte attorno alla fortuna bellica, il secondo è di carattere religioso, il terzo ed il quinto sono due testi teatrali incompiuti, il quarto è un sonetto per le nozze di Maria Giadrov e Pietro Zuliani, il sesto è un componimento lirico scritto in occasione della Pasqua, il settimo componimento contiene alcuni versi incompiuti, l'ottavo 
di manoscritti rilegati assieme in cartoncino, per la maggior parte scritti tra il 1817 e il 1818, dal titolo De Visiani. Scritti giovanili. Il contenuto del faldone, pur trattandosi sempre solo delle bozze degli scritti con correzioni e aggiunte a mano di de Visiani, si può dividere, grosso modo, in scritti compiuti (alcuni riportanti i titoli e altri senza titoli), e in scritti incompiuti presentati in forma di carte sciolte destinate a ritocchi, denotati con $D a$ compiersi e correggersi. Fanno eccezione i tre testi teatrali, incompiuti ma con i titoli apposti: Gli Adelfi di Terenzio (la traduzione dal latino della prima scena del primo atto della commedia Adelphoe di Publio Terenzio Afro), Simone e Sosia e Attilio Regalo Quartine composte estemporaneamente all'esame di Rettorica, datosi il 3 ag. alle 8 ore della mattina da Roberto de' Visiani, studente di Belle lettere.

Quanto al materiale intitolato contenuto nel faldone, esso si presenta come segue: Per l'immacolata concezione della SSma Vergine. Cantata di tre Pastorelle di Gerusalemme; In occasione delle faustissime nozze del sig: Pietro Zuliani colla gentilissima signora Maria Giadrov. In argomento d'estimazione. Sonetto; Per la Risurrezione di G. C. 8 sett. 1867; Domenica 4 gennaio 1818. Giorno della morte di Simon Sografi (incompiuto); Congedo dalla Dalmazia. Scritto in Poglizze nel maggio 1817 (il componimento è accompagnato da una lettera inviata a Niccolò Didos sotto il titolo Addio alla Dalmazia. Umiliato. Al Revmo Mons Don Niccolò Didos eletto Vicario Santo Capitolare della Metropolitana Primaziale Chiesa di Spalato. Rettore benemerito dell'Arcivescovile Seminario, Professore di Licenza Sacra nello stesso ec. ec. ec da Roberto de Visiani allievo del Seminario predefinito mentre passa all'l. R. Università di Padova per intraprendere gli studi medici); La giornata Autunnale (incompiuto). Tra i componimenti poetici compiuti, ma senza

componimento è il già menzionato autografo della poesia Congedo dalla Dalmazia, il nono componimento rappresenta alcune terzine senza titolo, segue poi la tragedia incompiuta Attilio Regolo con annottazione dell'autore "quartine composte per l'esame di retorica datosi ai 3 agosto alle ore 8 della mattina da Roberto de Visiani, studente di belle arti», una poesia di circa 600 versi incompiuti e una canzone anacreontica scritta in occasione del parto di Giulia Ferri. Cfr. Mika 1983, 427-438. Mika riporta però la segnatura sbagliata, non si tratta del faldone n. 23 come egli scrive, bensì del faldone n. 36. Quanto alla tragedia Attilio Regolo, è palese che de Visiani si sia ispirato all'omonimo melodramma di Pietro Metastasio, il che risulta evidente anche da alcuni versi che riporto in seguito: «O Roma, o Roma [...] Qual virtù non fiori fra muri tuoi / In seno a tue magnifiche contrade? [...] Ecco la moglie al pianto abbandonata / Con Pubblio Attilia segnala piagnente / Al piangere della madre addolorata / Non son già Attilio io più: Quello più volte / L'Africa fè tremar del fiero nome». 
titoli, meritano particolare attenzione i tre sonetti a pagina 5, 6 e 7 (quest'ultimo riporta la data 21 gennaio 1867), una poesia rivolta a Niccolò Tommaseo e una poesia composta da quattro quartine. L'ultima pagina dei manoscritti rilegati contiene l'elenco dei libri dati in prestito e di quelli avuti in prestito, con i nomi delle persone ed i titoli dei libri minuziosamente appuntati da de Visiani. Due sono i nomi che spiccano nell'elenco, entrambi sebenzani, Ferdinando de Pellegrini ${ }^{13}$ e Niccolò Tommaseo.

Considerando il fatto che de Visiani poeta è poco conosciuto, se non per pochi componimenti che videro la luce, come ad esempio Elegia per la morte di Francesco de' conti Ferri (Padova, 1823), In memoria di Francesco Sartori. Elegia indirizzata alla madre (Padova, 1849), II Bacio, pubblicato per le nozze Doro (1825), Il Salice, per le nozze De ManzoniFracanzani (1827) e la traduzione libera dallo spagnolo della poesia Nozze del garofano di Don José Selgas y Carasco (cfr. Pirona 1878-78, 663), mi sia permesso qui stilare i suoi primi esercizi di scolaro, per dirla con Tommaseo, conservando oltre ai testi leggibili e non coperti da macchie d'inchiostro, gli errori e la grafia originale. Si tratta per lo più di testi usciti dalla penna di de Visiani poco più che sedicenne, nati in gran parte sulla scia di quanto appreso in un ambiente scolastico stimolante, come fu il Seminario di Spalato, noto per la cura di alto livello degli studi retorici e classici. II Seminario spalatino, che vide tra i suoi migliori studenti Ugo Foscolo e Niccolò Tommaseo, lasciò un'impronta indelebile nel percorso di crescita professionale e privata del giovane de Visiani, che non dimentica di sdebitarsi con quanti gli avevano trasmesso conoscenze come Bernardino Bicego e Niccolò Didos. Li ricorda nella poesia Congedo dalla Dalmazia, la cui bozza, insieme alla minuta di una lettera che riporto qui per esteso, de Visiani aveva sottoposto al giudizio del suo professore:

Addio alla Dalmazia

Umiliato

Al Revmo Mons Don Niccolò Didos eletto Vicario Santo Capitolare della Metropolitana Primaziale Chiesa di Spalato. Rettore benemerito dell'Arcivescovile Seminario, Professore di Licenza Sacra nello stesso ec. ec. ec da Roberto de Visiani allievo del Seminario predefinito mentre passa all'I. R. Università di Padova per intraprendere gli studi medici

13 Sull'attività letteraria di Pellegrini, anch'egli discepolo del Seminario di Spalato, si veda Zorić 1955 e Zorić 2014. 
Chi non sa che le Muse furono sempre le consolatrici dell'uomo nelle sue più fatali disventure? Chi ignora avere un dolce sfogo un'anima veramente Poetica nell'esporre i suoi sentimenti nel divino linguaggio delle Muse? Benché la mia non sia tale, compenso, $\mathrm{M}$. Revdo. d'aver più volte avuta prova d'una cotal verità. Animato quindi da una tale lusinga anche nelle presenti cirsostanze egli è che ho tentato un tal mezzo, e forse non inutilmente del tutto. Ma siccome poi non tutti i metri a tutti i soggetti s'accordano, così mi servii di preferenza dello sciolto, come ritmo che per se stesso energico, e maestoso induce nell'anima una certa vibrazione, ed un certo entusiasmo, che forse più d'altra cosa dispone lo spirito all'allegrezza. Vorrà pertanto perdonarmi la gentilezza, e modestia di V. S. Rem. se seguendo un impulso irresistibile del mio cuore oso offrire al merito Vostro, ed alla Vostra gentilezza questo sforzo Poetico, qual ch'egli sia, certo che il Paterno animo Vostro non vorrà defraudarlo d'uno sguardo benigno animando con un sorriso di compatimento i giovanili miei sforzi. Mentre pertanto umilmente al bacio della sacra mano in inchino colgo l'onore di accertare Monsignoria la Vostra che più profondamente assai, che in fronte a queste rozze pagine sarà eternamente scolpito l'augusto Nome vostro nel cuore del più umile de' vostri Leni.

Congedo dalla Dalmazia

Scritto in Poglizze ${ }^{14}$ nel Maggio 1817

\footnotetext{
${ }^{14}$ Non credo si tratti di Poglizza vicino alla città di Almissa, località famosa per essere stata la capitale della Repubblica di Poglizza, una comunità dalmata sotto protettorato veneziano che riuscì a mantenere la propria autonomia dalla fine del Medioevo fino all'inizio dell'Ottocento. È molto più probabile che si tratti di una località fuori le mura della città di Sebenico che de Visiani spesso visitava in cerca di piante $\mathrm{i}$ cui nomi inserì nel suo Stirpium Dalmaticarum specimen. Si legge così, nell'opera citata, che Allium ascalonicum capillare, in illirico Ljutika, cresce «in saxosis agri Sibenicensis loco dicto Poglizze». Cfr. De Visiani, 1826, 24. Il toponimo Poglizze, ora scomparso, compare sulle mappe catastali austriache di Sebenico datate all'anno 1825 ben quattro volte (consultabile sul sito www.mapire.eu). Si tratta di una zona agricola che si trova a qualche chilometro dal quartiere cittadino Maddalena (in croato Mandalina) nella direzione sud-est, nonché di un'altra zona più a nord del quartiere Cernizza (in croato Crnica) e del Forte di San Giovanni (oggi Šubićevac). Sulla mappa austriaca del 1911 (Hafen und Rhede von Sebenico, Vodice e Zlarin, consultabile sul sito www. digitalna.nsk.hr) quella zona viene denominata Poljile. Il toponimo Poljice compare anche nell'elenco dei toponimi del comune catastale di Maddalena (Mandalina), si tratta di campi e vigne. Cfr. Blažević e Dorbić, 2016, 71. In più, anche la descrizione di Poglizze che de Visiani offre nel presente componimento supporta la tesi che si tratti di una località campestre:
} 
Semplice Villanel se astranio lido

Tragga repente, e le natie campagne,

E il caro aër natio lasci doglioso,

Sfogar gli è dolce per le meste lumi

„Di dolorosa nebbia il cor condenso"15,

Sfogo soave, e un amoroso Addio

Spinger sull'ali di sospiro ardente

Alle natie campagne, al Ciel nativo,

All'antro conscio di sue prime gioje,

All'arboscel, che d'agitevol vezzo

La stanca salma ricreò, che seco

Crebbe del pari, e sotto a cui talora

Carmi tentò di pastorenia musa.

Me pur, me pur dai patrj lari or lunge.

Orresistibil legge al bel Paese

Cui divide Appennin bacia il Tirreno

Tragge dolente d'Esculapio ${ }^{16}$ all'ara ${ }^{17}$.

Me pur le care piagge, e la diletta

Foce del Tizio romoroso, e questo

Non ignobile era giorno, or fido asilo

Di cortesia, teatralmente bella ${ }^{18}$

Mia Sebenico abbandonare m'astringe

Legge d'onor, che d'indugia nemica,

Tutti in me di Natura i più soavi

E tutti doni i ribellanti affetti

Sul conquiso cor mio scuote lo scettro.

E voi lasciar dovrò, tetti paterni,

\footnotetext{
«Mie delizie, amor mio, mia sola speme / Vaga Poglizze? lo te, rozza capanna / E voi piante, e te, selva, e te diletto, / Disfidator de' nembi [...]».

${ }_{15}$ Petrarca 1823, 90 (N. d. A.).

${ }^{16}$ Chiamato anche Epio, dio della medicina che acquistò perfetta cognizione delle virtù medicinali delle piante, per cui probabilmente attirò l'attenzione di de Visiani (N. d. A., si veda anche Fabre 1904, 58).

17 Se questa descrizione topica non le sembrasse esatta, mentre l'Italia è solo dalla parte d'Oriente circondata dal mar di lonio, mentre dall'Occidente confina col Golfo di Venezia, o Adriatico potrebbesi correggere così: Che Apennia parte, e cinge Adria, e Tirreno (nota di de Visiani). II giovane de Visiani si lasciò ovviamente influenzare dai famosi versi petrarcheschi: «il bel paese / ch'Appennin parte e 'I mar circonda e l'Alpe», Canzoniere, CXLVI, vv. 13-14 (N. d. A).

${ }^{18}$ Teatralmente bella, mentre Sebenico, posta sul pendio di un monte, presenta la figura d'un pezzo d'anfiteatro (nota di de Visiani).
} 
Voi, sotto a cui le prime aure di vita

Aspirar mi fu dato, e Te, più ch'altro ${ }^{19}$

Mie delizie, amor mio, mia sola speme

Vaga Poglizze? lo te, rozza capanna

E voi piante, e te, selva, e te diletto,

Disfidator de' nembi, amico acanto,

Cui confidai del giovenil mio plettro,

Misero! il primo suon?... Ma tu alfine,

Rimembranza crudel. Trattar che giova

Le ferite del cor, se d'un conforto

Recar non puossi almen misero ajuto.

Convien tutto lasciar, la patria, i campi,

I campi istessi. Alle velate antenne

Grave di nembi la rugosa fronte,

II mento antico, e le canute chiome

Spira Noto secondo, e già si scioglie

Ne siam già lungi, e solo vola in seno ${ }^{20}$

All'amiche campagne, ai tetti amici

Sull'ali del pensier la mente mia.

Dalmazia, e dove sei? In cupa avvolta

Caligine fatal già ti rapisti

Del tuo Figlio agli sguardi e teco insieme

La patria mia, teco Colei, che il freno

Delle Dalmate genti è giusto, e forte

Stringesi in mar, teco l'invitta un giorno

Reggitrice d'Illirio. O sempre illustre

E in guerra, e in pace, e degli Afinii afronte ${ }^{21}$,

E coi Re d'Oriente in te felici.

Spalato mia: sovra i sudati marmi,

Sull'Egizie colonne, onde se' bella

Stupido il ciglio il passeggero inarca,

${ }^{19}$ Questo aspirar per attrar l'aria ne' polmoni il Vocab. non I'ha, ma è bensì termine fisico (nota di de Visiani).

${ }^{20}$ Quel Ne potrebbe sembrare oscuro; ma se si rifletta che io parlo nel bollore della passione, e che quindi tutte le mie idee tender devono all'oggetto, che più la interessa, s'intenderà tosto ch'io parlo della patria, o della Dalmazia. E infatti non molto dopo io chiarifico l'idea dicendo: Dalmazia, e dove sei ec... (nota di de Visiani).

${ }^{21}$ Allor quando Salona sostenne per lungo tempo un accanita guerra co' Romani comandati da Afinio Pallione, benché poi vi cedesse. Sotto il numero plurale / maniera de' Latini / del Rè d'Oriente ognun vede ch'io parlo di Diocleziano ... (nota di de Visiani). 
Come selvaggio montanaro ammuta ${ }^{22}$,

Ove a real città tragga stupito.

Ma ben più assai, che le curvate, e spinte

Miracoli dell'arte, eccelse volte;

Ben più, che l'ardue, gigantesca mole

Che marmorea torreggia, e a se soggetta

Mira le pingui terre, i bei contorni,

Ed il fremente popolar tumulto,

Olimpo, al di cui pie mormora il nembo,

Me di Minerva il favorito accende.

Delubro, in mi sovra un medesimo altare

Han Scienza, e Virtù vittime incensi.

lo delle Muse fortunato asile, lo d'ogni bel saper culla felice,

Se pure io lascio, e in te gli eccelsi Genj

Che a gran sorte d'Illirio alberga

$\mathrm{O}$ ben sudati giorni, o ben sudate

Dolci fatiche! II memore pensiero

Su' que' prim' anni or mi trasporta, in cui

Alunno Tuo fui detto, e fù superbo

D'un tal nome il mio core, e sen compiaque.

Al fianco de' Tuoi Figli intente, e cheta

Sti buon lavor sulle vegliate carte

Vedea l'util Fatica, e sorridente

La giovine Speranza, e la salubre

Parsimonia robusta, e di soppiatto

Entrar talor il maturato Senno.

Religion Le prendeva aggiunta

Da candida Virtude, e comun cura

Era animar le giovinette penne

D'invitti sensi d'incorotto Vero.

Ahi! quegli anni felici or dove sono?

Chi reggerà nella temuta Italia

Il povero mio cor? qual certa norma

Qual prefiggermi io sol scorta fidata?

Nel tempestoso pelago de' mali,

Ond'innondato è l'universo e dove

Trovar fido sostegno, e quel severo

${ }^{22}$ Questa similitudine di Dante non serve ad altro, che ad esaltare l'idea dello stupore, ch'è certo più forte nel montanaro che nel passeggero (nota di de Visiani). 
Astro consolator lume di vita

Che il corso mio rischiari? un Didos dove?

Ah! tu soave d'ogni cor conforto,

Fè de' miei padri, il vacillante passo

Reggerai nell'agon. Nell'aspra guerra ${ }^{23}$

Dell'opre di quei Grandi, onde la schietta

Anima pura io contemplai, qualora

Crebbermi industri in giovinetta etade

Fresca memoria sosterrammi, ed opra

Prima del Ciel, che il Cielo è prima, e poscia

Degli alti di Virtude ineliti esempj

Di quell'Alme gentil sia dolce frutto.

Che più dunque tardar? Donisi alfine, E con coraggio, ai più temuti oggetti

Donisi al fine il doloroso Addio.

Ma prima scenda dall'eterna Soglia

Divino Amor; sul benedetto asilo

Della Dalmata speme apra e di schivar

D’ogni sua grazia inessicabil rio;

Nuovo fulgor sull'onorata fronte

Splenda raggiante dell'invitto Duce

Di sù bel fior di gioventù; novelle

Palme di gloria al maestoso crine

Gratitudine intrecci, e se le cure

Di Pastor tutte sul cor suo riposa,

Sacra Tiara, ben dovuto premio

A tanto Merto, sulla fronte augusta

D'improvviso splendor folgoreggiando

D'improvvisa allegrezza il volto avvivi

Alla dolente vedovata Chiesa.

Culti d'ogni saper, Genj, che l'alme ${ }^{24}$

Semplici ancora indirizzar godete

Sull'ardue di scienza inclite vie,

E di virtude, deh! Voi pur d'un core

\footnotetext{
${ }^{23}$ Se il pensiero, che la fresca memoria dell'opere virtuose de' miei Professori mi sosterrà sul retto sentiero, sembrasse espresso oscuramente, si può lumeggiare, al dir de' Pittori (nota di de Visiani).

24 In margine si legge: "A Bicego». I versi sono dedicati all'abate vicentino Bernardino Bicego che trascorse dieci anni a Spalato insegnando nel Seminario arcivescovile. Bicego, autore del poema I contorni di Spalato, è tra l'altro noto per essere stato professore del Tommaseo a Spalato (N. d. A).
} 
Che vostra cura fù, vostro pensiero

Accogliete, Voi pur questo, ch'io v'ergo

Dalla più bella delle belle valli,

Appiù d'un Colle lagrimoso Addio.

Di Poglizze li 30 Maggio 1817.

I 134 endecasillabi sciolti di de Visiani si possono suddividere in più unità tematiche, ognuna delle quali rappresenta un'autonoma esperienza personale, trasformata in immagine poetica: il congedo da Sebenico e da Spalato, l'addio al Seminario a ai professori, l'addio alla giovinezza in attesa di un avvenire incerto. L'alternarsi di tali immagini poetiche intrise di malinconia e del rimpianto di un addio, crea una forte impressione di mestizia, causata dalla imminente partenza dalla terra nativa, come il titolo stesso suggerisce. I versi riflettono gli interessi classici e romantici di de Visiani, che nei suoi sforzi di seguire i modelli poetici prestabiliti, si astiene da scorribande poetiche che potrebbero sembrare inappropriate o addirittura scorrette agli occhi di Didos. I riferimenti classici si evidenziano sia nelle scelte formali che in quelle di significato. Tutta la poesia si costruisce su una contrapposizione tra la vita passata, tranquilla e ingenua, e l'avvenire incerto che il nostro poeta deve affrontare da solo: "Chi reggerà nella temuta Italia / II povero mio cor? [...] Che il corso mio rischiari? un Didos dove?».

Ai motivi classici e romantici di de Visiani si alternano i motivi religiosi presenti in tutti i suoi componimenti giovanili, perfino nella poesia giocosa senza titolo ispirata alla passione esagerata dei dalmati per il cibo ${ }^{25}$, specialmente in occasione delle feste, vizio che spesso viene attribuito addirittura a San Girolamo. Le rime semplici di de Visiani, che riporto in seguito, sono intrise della felicità del giovane studente in attesa del prossimo viaggio a casa per le feste di Natale. I versi sono tanto più affascinanti in quanto rivolti al suo concittadino e compagno di scuola, Niccolò Tommaseo ${ }^{26}$, e rappresentanti, almeno per quanto mi è noto, dell'unico esempio di poesia giocosa rivolta al Dalmata illustre.

\footnotetext{
${ }^{25}$ In quella passione Enzo Bettiza, ad esempio, riconosce la passione che l'uomo prova per una donna: «Neppur l'attrazione per la donna, pur radicata profondamente nella sensualità tommaseiana dei dalmati, riusciva a superare quella per una pietanza gustosa». Cfr. Bettiza 1996, 213.

${ }^{26}$ Alla passione comune per il cibo sono dedicate alcune pagine dell'epistolario inedito Tommaseo - de Visiani, di prossima pubblicazione. Cito come esempio la lettera di Tommaseo a de Visiani, datata 3 gennaio 1868: «C. V. II mandorlato
} 
Niccolò sul tuo proposito

Sento dire, ch'ai pur fatto

Un grandissimo sproposito,

Solennissimo da matto.

lo nol credo in verità

Ma smentir, per carità

Or convien l'opinione

Di tantissime persone.

Voci vaghe non dicendo

Van dovunque disperdendo

Che ti sei ficcato in letto

Di tal rigido a dispetto

Per fuggire un po' di freddo

Per avere un po' di caldo

Fare cose da Goffreddo

Fare cose da Goccaldo.

Eppur sai, che vicinissime

Son le feste solennissime

Del santissimo Natale.

Preparato ha lo speziale

Lo speziale ha già impastato

Quel pasticcio prelibato

Soavissimo, e tenace

Che fin anco ai morti piace

E che usar si suole in queste

Benedette sante feste.

La mostarda è preparata

Che col pepe lavorata

Cogli arrosti più squisiti

Svegliar gli appettiti

E papa si suole in queste

Benedette sante feste.

Questo è il tempo pieno d'estro

è pianta che anco in Dalmazia per le feste di Natale ha la sua fioritura; ma non così bella come l'orto botanico tuo la porge. Il dono, pellegrino davvero, era degno d'essere inviato a Firenze; e i miei figliuoli fecero al soave ospite onorata accoglienza. Anch'io, quanto i denti consentivano, gli feci amore; e ritornare fanciullo mi piacque. Piaccia a Dio che ciò non accada per altra cagione. Grazie e auguri del tuo aff.

Tommaseo». Cfr. Biblioteca dell'Orto botanico dell'Università di Padova, Ar.B.13. 
Invocando il gran maestro

Il gentil m'esser appollo

Di gettarsi il plettro in collo

E riposati vicina

La piccante Maraschini

Ber cantare, e sol per queste

Benedette sante feste.

E tu ancor in letto stai

Sol fingendo mali, e guai?

Che via lascia quelle coltri

Ove tanto ancora poltri

Manda al diavol la Terzana ${ }^{27}$

Manda al diavol la Quartana

Vien da me, che beveremo

Vien da me, che scriveremo

E ciò tutto sol per queste

Benedette Sante Feste.

Di ispirazione cristiana è la cantata Per l'Immacolata Concezione Della SSma Vergine nella quale de Visiani riprende la storia dei genitori della Vergine, Anna e Gioacchino, narrata nell'apocrifo Protovangelo di Giacomo, aggiungendo però alcuni motivi tipici della letteratura dalmata dell'Ottocento quali le pastorelle e le ninfe. Il suo interesse per la botanica e per il mondo della natura emerge anche in questo componimento in cui l'ambientazione della storia si intuisce grazie alla scelta intenzionale delle piante menzionate, intrise di significati simbolici (la palma, il giglio ${ }^{28}$, il dittamo).

Per I'Immacolata Concezione

Della SSma Vergine

Cantata

Di tre Pastorelle di Gerusalemme

laele - Anna - Gedelta

I. Sù, sù parte vezzosa

Delle belle di Sion: oggi deggiamo

Il Sole prevenir, oggi la sposa

Di Gioacchin (che angelico messaggio

\footnotetext{
${ }^{27}$ Febbre, di solito malarica, come anche la quartana (N. d. A).

${ }^{28}$ Essendo simbolo di purezza e castità, il fiore è diventato il simbolo di Maria Vergine e si trova spesso nei dipinti che raffigurano l'Annunciazione.
} 
Mentir non può) grave rimane il seno

Di portentosa prole:

Gedelta, Anna, sù, sù, già spunta il sole.

G. E portentosa inver questa esser deve

Progenia benedetta,

Se a forza di prodigi

Fin nel materno sen viene concetta.

A. Ma intanto a Lei che rechevemo? II verno

Rugge d'intorno, e fra le brine, e il gelo

Un fior non s'alza ad abbellir suo stelo.

I. Non dir, Anna, così. Dove più folto

Di palme è il bosco io jeri un giglio scorsi,

Oh! Quanto vago. Lietamente in giro

Le dentellate a pina foglie gentili,

Su cui pareva timida disporsi

In macchiette sottili

Una languida porpora. Già il sole

Era per declinar; segno quel loco

A baciare quel fior mi chino umile

La greggia indi raccolgo, e vo' all'ovile.

lo quel vo' corre, e Le dirò: Quel Frutto,

Anna, che chiudi in seno anco immaturo,

Quel Frutto sia di questo fior più puro.

G. Fortunata laele. Anco mia Madre

Serba in chiuso giardino

Il dittamo adorato. lo vò far tanto,

Che fra' più vaghi rami

Mi doni quel, che più degli altri io brami.

lo quel vò darle, e Le dirò; quel Frutto

Che chiudi in sen sarà al celeste Amore,

Quale se avvien, che quella in copia coda

Il dittamo velluto alla ruggiada.

A. Ed io che far dovrò? Serbo gelosa

Tortore vaga da più mesi, cara

A Ninfe ed a Pastor. Questa recarle

lo voglio in dono, e Le dirò: La Prole,

Anna, che chiudi in sen, qual la vezzosa

Umile Tortorella

Sarà docile, e vaga, umile, e bella

I. Sù, sù, compagne

Prendiamo i doni:

Torniamo l'agne 
Al chuiso avil.

Vago, purissimo

Sia d'Anna il frutto;

Il giglio in tutto

Somiglierà.

G. Al ciel carissimo

Sia d'Anna il frutto;

Quest'erba in tutto

Somiglierà.

A. Umil, bellissimo

Sia d'Anna il frutto;

Mio dono in tutto

Somiglierà.

A3. Sù, sù, compagne,

Prendiamo i doni;

Torniamo l'agne

Al chiuso avil.

Fine

Alle carte stilate per lo più tra il 1817 e il 1818 troviamo uniti due sonetti di ispirazione religiosa, riportanti la data più recente; motivo per cui si giunge alla conclusione che de Visiani tenesse molto ai suoi scritti giovanili che serbava gelosamente e molto probabilmente correggeva e rivedeva tutta la sua vita. II primo sonetto, composto in età avanzata è nato in occasione della Pasqua del 1867 ed è ispirato alla vittoria di Cristo sulla morte.

Per la Risurrezione di G. C. 8 sett. 1867

Tra il sorriso, e la rabbia, a stretto viglia, Irrequieto il guardo unpur sereno, Morte con l'empia spada anco vermiglio

Sul sepolcro sedea del Nazareno.

Tutta degli angui l'orrida Famiglia Onde chiareggia il calvo teschio oscuro Mettea fischi, d'averno in culla figlio Schiumoso sornitando atro veneno. 
Quando repente spaventoso lampo

Esce $^{29}$ del marmo, e la codarda incalza

Che in Erebo $^{30}$ a cercar uno scampo.

Cupo suon di terror, di gioja misto

Mormora scotitor di balza in balza...

È il fremito d'Abisso, è sorto Cristo.

Oltre a questo sonetto, nelle carte di de Visiani ne troviamo altri tre senza titolo a pagina 5, 6 e 7 (quest'ultimo è accompagnato dalla data di composizione, 21 gennaio 1867).

Or che dell'arte, onde di $\mathrm{CaO}^{31}$ la fama

S'alza d'ogni altra al part lucida all'etra

Me pure accende irresistibil brama

A questo misto io ti sospendo a cetra

Del tuo vate tu qui dolente, e grama

Giaci, e se mai furtivo in sé penetra

Zeffiro amico, ali tu quel non v'impetra

Che l'alma immortale in te tanti anni.

Indi i soavi teneri momenti

Ove discorre il ricco Brenta d'aria

Sull'ali del più placido dei venti

Che pensando all'alta melodia

Godrà pur di piacer teneri momenti

L'accesa innamorata fantasia.

Traballa il monte e a mille in un baleno

Manda gli spettri a sgomentar Sionne

Stringo tremanti pargoletti al seno

L'atterrite di Giuda imbelli donne.

Alla scopa fatal già vengon meno

Del tempio l'inconcupe alte colonne

Squarciasi il velo ne v'ha cor sì sereno

Di cui fiero terrore or vero si indorne

\footnotetext{
${ }^{29}$ De Visiani aggiunse sopra «scoppia» (N. d. A.).

${ }^{30}$ Figlio del Caos e della Notte. Fu cangiato in primo fiume d'inferno per aver soccorso i Giganti. Cfr. Fabre 1904, 57.

${ }^{31}$ Ant. $\operatorname{caos}$ (N. d. A.).
} 
In neve bende avvolge l'aureo crine Febo $^{32}$ oscurato e in un confusi e misti Minaccian gli elementi alte rovine.

Del suo nume ai sospir flebili e tristi Par che ogni sasso anco a pietà s'incline, In sol, duro mio cor, tu sol resisti.

Sonetto fatto li 21 Gennajo 1867

Tempo fu già, che, posta in cieco obblio Sua divina Cagion, mortale altero Numi far mossi, e d'un infame un Dio Poco stette a crearsi uman pensiero.

Ma poiché scese a riscattare il rio Degli error suoi già inorgoglito e fero Dell'Eterno il gran Figlio, allor svanio Di sì rei simulacri il lungo impero.

Fede pura successe, e costo ardore Nuda schiettezza, ed anni stà verace E dell'uomo tutto ricercavo il cuore.

Or che vil frode, a simulato ingegno Torna de' cori a minacciar la pace De' prischi Dei di vena tornato il Regno?

Pur essendosi azzardato in diverse forme letterarie, è evidente che la forma preferita di de Visiani sia il sonetto e che le sue rime, prevalentemente di carattere religioso, nascano in base ad un inventario prestabilito di figure e strumenti retorici, fatto che li rende poco originali e poco comprensibili, se non ad un lettore appassionato di studi classici e retorici. Anche il linguaggio poetico presente nei sonetti, troppo gravato di latinismi e di reminiscenze classiche, a volte risulta essere involuto e difficilmente afferrabile, cosa che non si può dire per l'unico

${ }^{32}$ Cioè Apollo, il dio della musica, della poesia, delle arti e della medicina (N. d. A.). 
sonetto encomiastico trovato tra le carte di de Visiani, composto in occasione delle nozze di Maria Giadrov ${ }^{33}$ e Pietro Zuliani ${ }^{34}$.

In occasione

Delle Faustissime Nozze

Del sig: Pietro Zuliani

colla gentilissima Signora

Maria Giadrov

In argomento d'estimazione

Sonetto

Unir maschia saggezza a nobil core

Che di non basse idee nutre il pensiero;

Semplicitade unir a bel candore.

Di giovanile età fregio primiero.

Unir d'alta beltà candido fiore

A cor, che solo di Virtude è altero;

A Pietro con gentil lancio d'Amore

Unir Maria, che ha del suo cor l'impero.

Ah! che non è d'Amore do' pena già questa.

Virtù fu certo, che si chiari spirti

D'indissolubil nodo avvinse e unio.

È Virtù che la fece ora s'appresta

Illustri sposi, e gli amorosi mirti

Che non puote cotanto un cieco Dio.

Nel sonetto sopra riportato si rispecchia il tentativo del poeta di rendere omaggio non solo agli appartenenti dei due illustri casati sebenicensi,

\footnotetext{
${ }^{33}$ La famiglia Giadrov diede i natali a numerose personalità importanti per la storia di Sebenico, quali ad esempio il medico Vincenzo Giadrov, che pubblica a Milano nel 1831 l'opera Virtù antifebbrile della gomma rasina di olivo, resultate dalle sperienze e il mercante Andrea Giadrov, marito della sorella della nonna paterna di Niccolò Tommaseo la cui casa a Sebenico ospitava il negozio del padre di Antonio Marinovich, amico di Tommaseo che muore giovanissimo nel 1834 ed al quale Tommaseo dedica il romanzo epistolare Dell'animo $e$ dell'ingegno di Antonio Marinovich, pubblicato a Venezia nel 1840.

34 Pietro Zuliani, farmacista a Sebenico, nel 1864 membro del comitato per la costruzione del Teatro Mazzoleni a Sebenico, fu discendente di un illustre casato sebenicese. Si suppone a lui appartenesse il palazzo Divnić a Sebenico.
} 
fatto importante in quanto ulteriore testimonianza della stima e dell'affetto che legava de Visiani alla sua città natale ed ai suoi abitanti ai quali era unito da vincoli sentimentali, ma rappresenta nello stesso tempo anche una lode all'Amore romantico che, per lui che dedicò la sua vita alla scienza, ormai diventa un archetipo irrealistico e irraggiungibile.

\section{Conclusione}

Come accennato in precedenza, pur trattandosi di scritti che possiamo definire compiuti, non bisogna trascurare il fatto che de Visiani, se avesse deciso di pubblicarli, li avrebbe rivisti e controllati, forse anche sottoposti al giudizio dell'amico Tommaseo. Sono perciò incline ad attribuire alcune irregolarità stilistiche dei testi alla mancata revisione degli scritti che forse non erano mai stati destinati alla pubblicazione. Anche se la migliore espressione letteraria di de Visiani si trova nelle sue opere di prosa scientifica, i suoi scritti giovanili, oltre a tracciare un lato biografico meno noto dell'illustre botanico, hanno senza dubbio un valore estetico e come tali meritano di essere sottoposti al giudizio della critica che, ne sono sicuro, prenderà in considerazione la complessità dei tempi e le circostanze in cui sono nati.

\section{Bibliografia}

Bettiza, Enzo. Esilio. Milano: Mondadori, 1996

Blažević, Milivoj e Dorbić, Boris. Hrvatsko nazivlje zemljišta i krajobraza obalnog dijela šibenskog područja od 18. do 20. stoljeća in "Osvit», Šibenik: Ogranak Matice hrvatske u Šibeniku, 2016., pp. 58 - 86

De Visiani, Roberto. Stirpium Dalmaticarum specimen, Patavii: Typis Crescinianis, 1826

De Visiani, Roberto. Una corsa botanica sul battello a vapore lungo la costa della Dalmazia, "Strenna dalmata», 1847, pp. 81 - 87

Digitalne zbirke Nacionalne i Sveučilišne knjižnice u Zagrebu: www.digitalna.nsk.hr

Fabre, Alessandro. Dizionario della mitologia greca e romana, Torino: Libreria Salesiana, 1904

Habsburg Empire - Cadastral maps (XIX. century): www.mapire.eu

Grubišić, Slavo. Visiani i rodni grad Šibenik in Zbornik Roberta Visianija Šibenčanina, Šibenik: Muzej grada Šibenika, 1983., pp. 27 - 45

Knežić, Boško. "Oh, mio caro fratello di latte» - Paolo Mazzoleni i Niccolò Tommaseo. «Fluminensia», XXVIII, 1, Rijeka, 2016., pp. 197 - 209

Mazzoleni, Paolo. All'illustrissimo reverendissimo monsignor vescovo Antonio Giuseppe Fosco questo ricordo dell'inaugurazione del monumento sepolcrale a Roberto de Visiani seguita il 10 luglio 1880 nel cimitero di Sant'Anna in Sebenico alle pietose sue cure dovuta e coll'esequie da lui celebrate resa più solenne in segno di riconoscenza affettuosa Paolo 
Mazzoleni e Luigi D.r Zuliani dedicano riverenti, Sebenico: Tip. eredi S. Anich, 1880

Mazzoleni, Paolo. Il primo centenario della nascita del professore dott. Roberto de Visiani, Conferenza tenuta la sera del 3 marzo 1900 nella sala maggiore della secolare Società del Casino di Sebenico, Zara: Stab. Tip. di S. Artale, 1900

Mika, Cvjetko. Robert Visiani kao jezikoslovac i pjesnik in Zbornik Roberta Visianija Šibenčanina, Šibenik: Muzej grada Šibenika, 1983., pp. 427 - 443

Notolini, Giulia. L'archivio dell'Orto botanico di Padova e dei suoi prefetti (17631921): inventario analitico, vicende istituzionali e profili biografici, Università Ca' Foscari Venezia, 2017-2018

Petrarca, Francesco. Canzone XIII in Rime di Francesco Petrarca, col commento di G. Biagioli, volume primo, Milano: Per Giovanni Silvestri, 1823

Pirona, Giulio Andrea. Della vita scientifica del professore Roberto de Visiani. Commemorazione del m. e. Prof. Giulio Andrea Pirona in Atti del Reale Istituto Veneto di Scienze, Lettere ed Arti, dal novembre 1878 all'ottobre 1897, Tomo V, Serie V, Venezia: Tip. di G. Antonelli, 1878 - 79, pp. 637 - 673

Stošić, Krsto. Javna dobrotvornost u Šibeniku in Stjepan Sirovica. Iz prošlosti Šibenika i Dalmacije. Razdoblje od 18. do 20. stoljeća, Zagreb: Medicinska naklada, 2006., pp. 7 - 31

Tomas, Valter. Strenna Dalmata (1847.). Prvi dalmatinski novogodišnji almanah, Zadar: Sveučilište u Zadru, 2018.

Tommaseo, Niccolò. Diario intimo, a cura di Raffaele Ciampini, Torino: Einaudi, 1938

Zorić, Mate. Sjenovita dionica talijanske književnosti. Romantički pisci u Dalmaciji na talijanskom jeziku, uredile Nedjeljka Balić-Nižić i Sanja Roić, Zagreb: Hrvatska sveučilišna naklada, 2014.

Zorić, Mate. Ferdinando Pellegrini - prevodilac naših narodnih pjesama in Zbornik instituta za historijske nauke u Zadru, Zadar, 1955., pp. 145 - 164

\section{RANI RADOVI ŠIBENČANINA ROBERTA DE VISIANIJA}

$\mathrm{U}$ radu se donosi uvid u prve mladenačke uratke slavnog botaničara svjetskog glasa, Roberta de Visianija, koji se čuvaju u Povijesnom arhivu Botaničkog vrta u Padovi u mapi br. 36, sveščić br. 5. Riječ je o neobjavljenim rukopisima, nastalim uglavnom između 1817. i 1818., sašivenim u kartonski omot i naslovljenim De Visiani. Scritti giovanili. Sadržaj mape se može podijeliti na završene uratke od kojih su neki naslovljeni, a neki bez naslova, kao i na nedovršene uratke koji su predviđeni za doradu i koje pronalazimo na nenumeriranim listovima. $U$ radu je pažnja usmjerena uglavnom na završene čitke tekstove. Radi se o prigodnim uradcima koji su pisani u skladu s poetikom vremena u kojem su nastali.

Ključne riječi: Roberto de Visiani, Scritti giovanili, Povijesni arhiv Botaničkog vrta u Padovi 